\title{
The Third American Society of Regional Anesthesia and Pain Medicine Practice Advisory on Local Anesthetic Systemic Toxicity Executive Summary 2017
}

\author{
Joseph M. Neal, MD, * Michael J. Barrington, MBBS, FANZCA, PhD, † Michael R. Fettiplace, MD, PhD, $\$$ \\ Marina Gitman, MD, \& Stavros G. Memtsoudis, MD, PhD,// Eva E. Mörwald, MD,// \\ Daniel S. Rubin, MD, ** and Guy Weinberg, MD\$††
}

\begin{abstract}
The American Society of Regional Anesthesia and Pain Medicine's Third Practice Advisory on local anesthetic systemic toxicity is an interim update from its 2010 advisory. The advisory focuses on new information regarding the mechanisms of lipid resuscitation, updated frequency estimates, the preventative role of ultrasound guidance, changes to case presentation patterns, and limited information related to local infiltration anesthesia and liposomal bupivacaine. In addition to emerging information, the advisory updates recommendations pertaining to prevention, recognition, and treatment of local anesthetic systemic toxicity.
\end{abstract}

What's New in This Update? This interim update summarizes recent scientific findings that have enhanced our understanding of the mechanisms that lead to lipid emulsion reversal of LAST, including rapid partitioning, direct inotropy, and post-conditioning. Since the previous practice advisory, epidemiological data have emerged that suggest a lower frequency of LAST as reported by single institutions and some registries, nevertheless a considerable number of events still occur within the general community. Contemporary case reports suggest a trend toward delayed presentation, which may mirror the increased use of ultrasound guidance (fewer intravascular injections), local infiltration techniques (slower systemic uptake), and continuous local anesthetic infusions. Small patient size and sarcopenia are additional factors that increase potential risk for LAST. An increasing number of reported events occur outside of the traditional hospital setting and involve non-anesthesiologists.

(Reg Anesth Pain Med 2018;43: 113-123)

nterventions involving local anesthetics are ubiquitous throughout medicine and are performed by anesthesiologists, other physicians, dentists, and paramedical personnel. Local anesthetic systemic toxicity (LAST) remains a serious adverse event despite advances in prevention, detection, and treatment. Such events range across a continuum from mild subjective prodromal symptoms to seizure, cardiac arrest, and/or death. As noted in our previous

From the *Virginia Mason Medical Center, Seattle, WA; †St Vincent's Hospital, University of Melbourne, Melbourne, Australia; †Massachusetts General Hospital, Boston, MA; §College of Medicine, University of Illinois, Chicago; ||Hospital for Special Surgery, Weill Cornell Medical College, New York, NY; **University of Chicago; and $\dagger \dagger$ Jesse Brown VA Medical Center, Chicago, IL.

Accepted for publication November 15, 2017.

Address correspondence to: Joseph M. Neal, MD, 1100 Ninth Ave (B2-AN), Seattle, WA 98111 (e-mail: Joseph.Neal@virginiamason.org).

G.W. is an officer, director, shareholder and paid consultant of ResQ Pharma, Inc. He also created and maintains www.lipidrescue.org, an educational web site. The authors otherwise declare no potential conflict of interest.

This article discusses an off-label use of lipid emulsion (eg, Intralipid) for treatment of local anesthetic systemic toxicity.

Copyright (C) 2018 by American Society of Regional Anesthesia and Pain Medicine

ISSN: $1098-7339$

DOI: 10.1097/AAP.0000000000000720 practice advisory, "a variety of factors influence the likelihood and severity of LAST, including individual patient risk factors, concurrent medications, location and technique of block, specific local anesthetic compound, total local anesthetic dose (the product of concentration $\mathrm{x}$ volume), timeliness of detection, and adequacy of treatment." The overall rarity of LAST and the fact that episodes can occur despite proper technique argue strongly for education and preparedness among all professionals who administer local anesthetics to their patients.

The American Society of Regional Anesthesia and Pain Medicine (ASRA) convened its first Conference on Local Anesthetic Toxicity in 2001 coinciding with initial clinical experience after release of the levo-enantiomers ropivacaine and l-bupivacaine, plus emerging laboratory observations on the effectiveness of lipid emulsion as an antidote for LAST. In 2008, a second advisory panel met to develop a rational approach for treatment ${ }^{2}$ and to assimilate information on the history, mechanisms, prevention, and detection of LAST. ${ }^{1,3-7}$ Consistent with ASRA's commitment to update its practice advisories as new knowledge emerges, in 2015 , the board of directors commissioned a small group of current researchers to develop this third advisory. In compliance with calls from medical journal editors to focus practice advisory updates on new information, ${ }^{8}$ we herein present developing knowledge on the mechanisms of lipid resuscitation, new insights into incidence and epidemiology, evolving patterns of case presentation, and revised treatment protocols.

This executive summary condenses information and recommendations from supporting articles that have been published recently ${ }^{9}$ or concurrently as part of this practice advisory. ${ }^{10-13}$ Practitioners are encouraged to read these articles to understand the basis underlying our recommendations.

\section{METHODS}

Individual supporting articles provide details relevant to the sources accessed to derive their analysis and recommendations. In general, standard medical literature search engines and article cross-referencing were used to identify animal and human studies, case reports and series, and epidemiologic information. The focus was on new publications and developments since our 2010 advisory.

The panel consisted of recognized experts in LAST and/or guideline development and includes the authors of this article. The ASRA provided standard travel reimbursement for members of the advisory group who met in Chicago on March 26, 2016. No panelist was paid for their participation, nor did ASRA receive external funds to support the advisory process.

The second ASRA practice advisory sought input from external sources including other anesthesiologists with expertise in LAST, professional organizations representing physicians and dentists who commonly use local anesthetics, and open forum 
discussion of our recommendations. ${ }^{1}$ Because the current practice advisory represents an interim update and does not recommend major changes to treatment, external input was solicited only at open forum presentations coinciding with the ASRA 2015 and 2016 spring meetings.

\section{Strength of Recommendations}

As stated in our 2010 advisory: "There are no randomized clinical trials (RCTs) evaluating serious human LAST; future RCTs are unlikely because of the rarity of these complications and the associated difficulty of obtaining informed consent for medical interventions in critical illness. Common strength-of-evidence schemas that are based on RCT-level evidence are therefore inappropriate for the topic of human LAST, but may be appropriate for animal studies. Hence, the panel's recommendations are based on a modified Classification of Recommendations and Levels of Evidence schema that was developed by the American College of Cardiology/American Heart Association ${ }^{14}$ (Table 1). The panel wishes to emphasize that assigning a level of evidence $\mathrm{B}$ or $\mathrm{C}$ should not be construed as implying that the associated recommendation is supported by conflicting data or is limited by conflicting interpretations of the available data. Rather, such recommendations reflect our recognition of the importance of the particular question as it relates to LAST and to the reality that the specific question is either yet to be addressed by an RCT or does not lend itself to experimental inquiry in humans."

\section{Limitations}

As with previous ASRA advisories, "readers of this manuscript are reminded that practice advisories are created when data on a subject are limited or non-existent. Advisories rely on limited clinical and animal data and, as such, the synthesis and interpretation of data by one group of experts may differ from conclusions by

TABLE 1. Classification of Recommendations and Levels of Evidence

\section{Classification of Recommendations}

Class I Conditions for which there is evidence and/or general agreement that a given procedure or treatment is useful and effective

Class II Conditions for which there is conflicting evidence and/or a divergence of opinion about the usefulness/efficacy of a procedure or treatment IIa. Weight of evidence/opinion is in favor of usefulness/efficacy

IIb. Usefulness/efficacy is less well established by evidence/opinion

Class III Conditions for which there is evidence and/or general agreement that the procedure/treatment is not useful/effective, and in some cases may be harmful

\section{Level of Evidence}

Level A Data derived from RCTs

Level B Data derived from nonrandomized or laboratory, eg, animal, studies; supported by multiple case reports or case series

Level C Consensus opinion of experts

Table 1 is presented unchanged from the Second ASRA Practice Advisory on LAST ${ }^{1}$ and is modified from an American College of Cardiology/American Heart Association schema for developing and grading guidelines. ${ }^{14}$ another set of equally qualified experts. Thus, practice advisories represent a level of recommendation less than that offered by standards or clinical practice guidelines. The recommendations contained herein do not define standard of care. They are not intended to replace clinical judgment as applied to a specific patient scenario. These recommendations are intended to encourage optimal patient care, but cannot ensure the avoidance of adverse outcomes. As with any practice advisory recommendation, these are subject to revision as knowledge of specific complications advances."15,16

\section{DISCUSSION}

\section{Mechanisms Underlying Lipid Resuscitation}

The most important recent advances in our knowledge of LAST relate to the basic science of lipid emulsion resuscitation, within both anesthesiology and other disciplines that involve medical toxicology. New to this practice advisory, Fettiplace and Weinberg ${ }^{10}$ provide an in depth discussion of the mechanisms that underlie the therapeutic reversal of LAST by lipid emulsion. Henceforth, we summarize the most salient points of this topic, the complexity of which necessitates reading the accompanying supporting article to move beyond basic understanding.

Lipid emulsion reversal of LAST is ultimately linked to cellular mechanisms that are affected by local anesthetics. Under normal circumstances, local anesthetics block nerve conduction by inhibiting transduction of sodium, calcium, and potassium through voltage-gated ionotropic channels located in the cell membrane. Acute local anesthetic cardiotoxicity negatively impacts myocardial contractility, cardiac conduction, and systemic vascular resistance through a complex and widespread set of events that involve channel blockade, metabolic signaling, and intracellular energy production, that is, inhibition of oxidative phosphorylation. The clinical effects of these cellular events manifest initially as hypertension and tachydysrhythmias that progress to depressed cardiac conduction and performance (reduced cardiac output), bradycardia, and hypotension. Similarly, local anesthetic toxic effects on central nervous system (CNS) ionic channels manifest initially as altered mental status and/or mild prodromal symptoms such as paresthesias, tinnitus, and agitation and progress to seizure and possible coma.

Lipid emulsion resuscitation was first proposed as a treatment for LAST in 1998 and translated into clinical practice 8 years later. ${ }^{10}$ Over this timeframe, a number of potential mechanisms of action were proposed, the most prominent of which involved the hypothesis that lipid emulsion infusion effectively created an intravascular lipophilic "sink" into which lipid soluble local anesthetics such as bupivacaine partitioned and were ultimately removed from the body. ${ }^{17}$ Twenty years later, lipid emulsion therapy is believed to involve multiple mechanisms that involve active shuttling of local anesthetic away from the heart and brain, cardiotonic effects that involve the heart and/or vasculature, and postconditioning cardioprotective effects. ${ }^{10}$

\section{Shuttling Effects}

Rather than acting as a static lipid sink, current research supports the concept that lipid emulsion works as a dynamic carrier to scavenge local anesthetic away from high blood flow organs that are most sensitive to LAST (ie, the heart and brain) and redistribute it to organs that store and detoxify the drug (ie, muscle and liver, respectively) ${ }^{18}$ (Fig. 1). The precise mechanisms of local anesthetic binding to lipid droplets are not understood fully but are believed to combine thermodynamic effects, for example, electrostatic attraction and physicochemical characteristics such as lipophilicity and acid-base ionization, as positively charged 


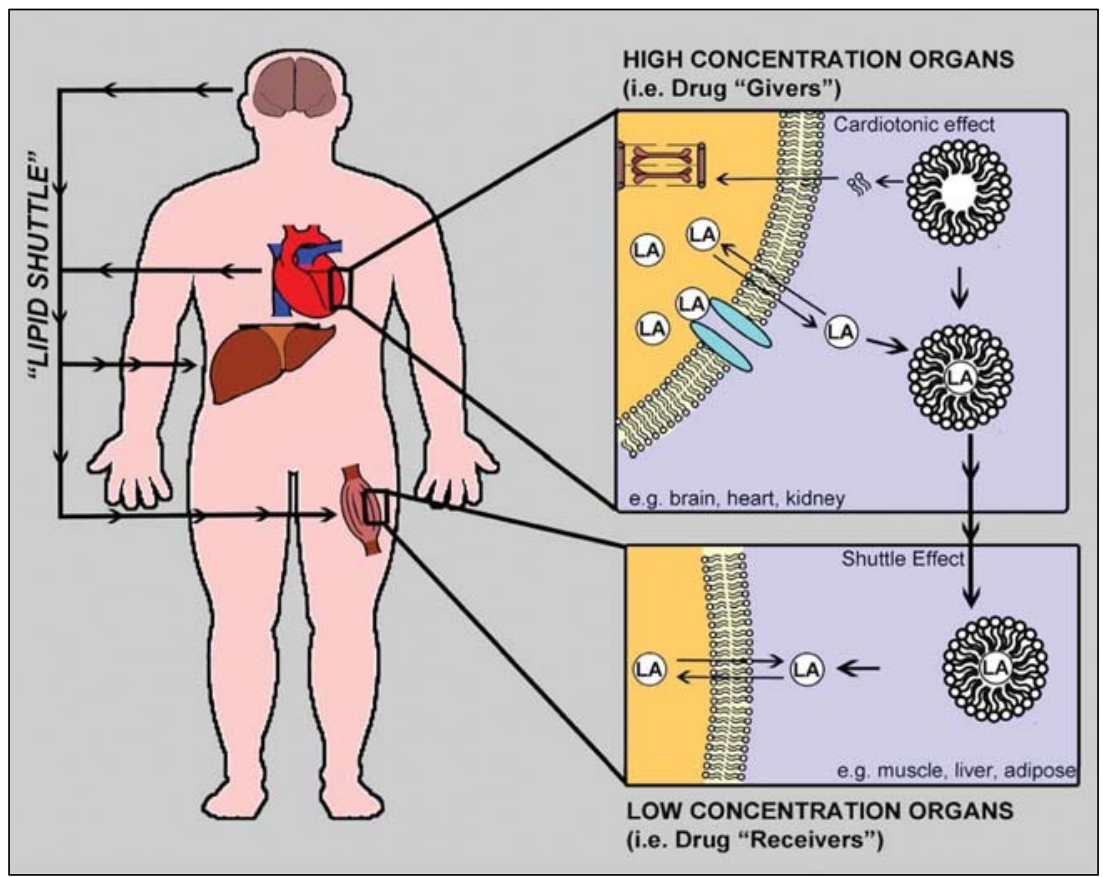

FIGURE 1. Schematic representation of mechanisms associated with lipid emulsion reversal of LAST, including shuttling of lipophilic local anesthetic from the heart and brain to muscle and liver. Adapted from Fettiplace et al ${ }^{18}$ with permission.

fat-soluble local anesthetic molecules bind to negatively charged lipid particles. These observations appear to support lipid emulsion having greater efficacy in shuttling the more lipophilic local anesthetics such as bupivacaine. Nevertheless, even the less lipophilic local anesthetics such as lidocaine or mepivacaine are highly lipid soluble and carry a positive charge at physiologic $\mathrm{pH}$. Therefore, lipid emulsion should be effective at reversing toxicity after overdose with lidocaine ${ }^{19}$ or mepivacaine. Further evidence suggests that together these pharmacokinetic attributes work to accelerate redistribution of local anesthetics by increasing the $\alpha$ half-life in whole blood, while decreasing the concentration of local anesthetic in the nonlipid fraction. The net effect shortens local anesthetic elimination half-life. ${ }^{20-22}$ Lipid emulsion also has nonscavenging effects that manifest as cardiotonic and postconditioning effects.

\section{Cardiotonic Effects}

Several lines of evidence support the concept that lipid increases cardiac performance, which enhances the shuttling effect. The direct cardiotonic effects of lipid emulsion increase cardiac contractility, which increases cardiac output and blood flow through affected organs. Volume loads associated with lipid resuscitation improve cardiac function by a simple preload effect, although this effect is significantly less than the positive inotropy seen in both intact rat and isolated heart models during infusion of lipid. Lipid infusion also increases blood pressure via poorly understood effects on the peripheral vasculature. Together, these mechanisms serve to improve both cardiac output and blood pressure. ${ }^{23,24}$

\section{Postconditioning Effects}

Recent laboratory experiments support the concept that adverse cellular effects of LAST overlap with mechanisms of cardiac ischemia-reperfusion injury. ${ }^{25}$ Coupled with the observation that infused lipid emulsion activates cardioprotective pathways, this provides an additional mechanism of postconditioning benefit to the local anesthetic-toxic heart. ${ }^{26,27}$ The explanations behind these purported benefits are discussed in detail in the supporting article. ${ }^{10}$

\section{Incidence and Epidemiology}

Since publication of our 2010 practice advisory, new evidence has furthered our understanding of LAST epidemiology. This information is derived from analysis of administrative databases, registries, and case reports/case series. Of particular importance is publication of several studies that suggest the incidence of LAST associated with epidural ${ }^{28}$ and peripheral nerve blocks (PNBs) is decreasing, ${ }^{29}$ which has led some experts ${ }^{29,30}$ to debate whether or not LAST remains a clinically relevant complication.

\section{Administrative Databases}

One avenue to assess the frequency of LAST in the general community population is to query large administrative databases such as the Premier Perspective Database (PPD). ${ }^{31}$ As part of the current advisory series, Mörwald et $\mathrm{al}^{9}$ recently presented data from over 400 hospitals on nearly 238,500 patients who received a PNB for total joint arthroplasty between 2006 and 2014. This methodology's weakness is that it lacks clinical details beyond those revealed by diagnostic or billing codes, and because no specific coding for LAST could be identified in the hospital reports, the methodology relies on surrogate markers such as seizure, cardiac arrest, or administration of lipid emulsion. Although the occurrence of seizure or cardiac arrest may overestimate the frequency of LAST, the increased use of lipid emulsion on the day of surgery is likely specific to the true diagnosis. In Mörwald et al's ${ }^{9}$ study, the cumulative rate of LAST was $0.18 \%$, that is, the rate of patients receiving a PNB and experiencing at least one LAST-associated outcome was $1.8 / 1000$. During the 9-year study period, the overall adverse outcome rate trended downward without the trend reaching statistical significance, whereas the use of lipid emulsion increased 
significantly. The investigators concluded that, although this population-based frequency of LAST is low, it should be considered clinically meaningful.

The National Inpatient Sample (NIS) contains a diagnostic code specific to LAST, although it remains uncertain how specific the coding is for LAST events, because errors in coding can occur as a result of physician documentation or data abstraction. In the current practice advisory series, Rubin et $\mathrm{al}^{12}$ report an incidence of LAST intermediate between that of the PPD and those of single institutions and specialized registries. Their database query of over 710,000 PNBs placed for total joint arthroplasties revealed an average adjusted incidence of 1.04 (confidence interval [CI], 0.49-1.80) LAST events per 1000 PNBs over the 15-year period 1998 to 2013. Of these events, 1 in 5 was considered major, that is, involved a seizure $(8.1 \%)$ or a major cardiac complication $(6.8 \%)$. Contrary to observations from case reports, ${ }^{4}$ there were no deaths and multivariable analysis found no correlation between LAST and age or comorbidity. Total shoulder arthroplasty was associated with LAST more often than knee or hip operations (odds ratio [OR], 4.35; CI, 1.96-9.65), as was being cared for in a large to medium-sized hospital. Over the 15-year study period, and similar to Mörwald et al's ${ }^{9}$ findings, the odds of a LAST event trended downward by $10 \%$ per year (OR, 0.90; CI, 0.84-0.96) despite increased PNB use.

The PPD's 1.8/1000 cumulative rate and the NIS 1.04/1000 incidence, while similar, are considerably higher than the 0.04 / 1000 to $0.8 / 1000$ LAST incidences reported contemporaneously from various registries, ${ }^{32}$ national surveys, ${ }^{33}$ and single institutions. ${ }^{29,34}$ Variations in the incidence may reflect differing definitions of LAST. For example, studies that include generalized CNS excitation ${ }^{35}$ or minor cardiac complications ${ }^{12}$ in addition to seizure and cardiac arrest report a higher incidence of LAST $(0.87 / 1000$ to $1.04 / 1000)$ than studies ${ }^{29}$ that only include seizure or cardiac arrest $(0.04 / 1000)$. With regard to expertise and caseload, PPD data suggest that LAST events within the community, where case volumes may be low and ultrasound use not universal, may be more common than those reported from registry instruments or single institutions that manifest expertise in regional anesthesia. Conversely, the NIS study found no difference between teaching and nonteaching hospitals. In summary, although administrative databases have specific weaknesses related to the lack of relevant clinical information on individual LAST events, they do demonstrate that LAST remains a rare yet relevant issue in the community at large.

\section{Registries}

A wealth of registry and observational study data have been published since 2010. These works range from extensions of previously published quality assurance data sets, ${ }^{36,37}$ national databases $^{33,38}$ and international registries, ${ }^{35,39}$ to specific registries that focus on pediatric practice, ${ }^{40-42}$ single-center data, ${ }^{29,32}$ and data specific to interscalene brachial plexus block. ${ }^{34}$

The most important new finding from the Australian and New Zealand Registry of Regional Anaesthesia (now the International Registry of Regional Anaesthesia) is that ultrasound guidance reduces the risk of LAST throughout its continuum by $60 \%$ to $65 \%$ (propensity analysis OR, 0.35-0.36) as compared with peripheral nerve stimulation alone. ${ }^{35}$ This observation is supported by the Dartmouth registry, which reported only 1 seizure in 12,668 ultrasound-guided blocks. ${ }^{32}$ The International Registry of Regional Anaesthesia registry also reported a surprisingly higher risk of LAST with lidocaine than with ropivacaine, which the authors speculated may reflect a practitioner's false sense of security when using relatively less toxic lidocaine. ${ }^{35}$
National survey data from Italy ${ }^{38}$ reveal an incidence of LAST during PNB of 0.34/1000 blocks, and Finnish data ${ }^{33}$ document a similar 0.37 per 1000 PNBs. Of note, the Finnish incidence is lower if all LAST events (including epidural, but not spinal, anesthetics) are included (0.07 per 1000). This observation is consistent with previous knowledge that LAST is 4 to 5 times more common with PNBs than with epidurals. ${ }^{1}$ If one were to combine LAST events reported since 2010 from 11 registries and observational studies, the population frequency is $0.27 / 1000$ (95\% CI, 0.21-0.35) (69 events per 251,325 PNBs). ${ }^{11}$

Data from observational studies offer some insights into whether or not experience and expertise affect the rate of LAST. The Finnish study ${ }^{33}$ noted a 3.3 relative risk of having a LAST event in a nonuniversity hospital as compared with a university hospital. Similarly, the Hospital for Special Surgery in New York, which represents a specialized high-volume regional anesthesia practice, reported 0.04 seizures per 1000 blocks, which is lower than other reports and may not apply to the community at large. ${ }^{29}$

Pediatric anesthetics captured in registries ${ }^{40,42}$ and singlecenter $^{41}$ observational studies confirm a very low overall rate of LAST in children. Data from the 2012 Pediatric Regional Anesthesia Network documented no LAST episodes in 14,917 procedures, ${ }^{42}$ although its most recent analysis of over 100,000 regional anesthetics will report 7 severe LAST episodes (cardiac arrest or seizure) $-0.076 / 1000,95 \%$ CI, 0.029 to $0.14 / 1000$ (J.M. Neal, $\mathrm{MD}$, and D.M. Polaner, MD, personal written communication September 19, 2017). The French-Language Society of Pediatric Anaesthesiologists' registry ${ }^{40}$ reported rates of $0.16 / 1000$ for PNBs and 1.3/1000 neuraxial blocks (excluding spinal anesthesia). The frequency of LAST associated with pediatric continuous catheter techniques is higher, 1.53 per $1000 .{ }^{41}$ When compared with the overall population frequency of $0.27 / 1000$ (95\% CI, $0.21-0.35),{ }^{11}$ it can be argued that the rate of LAST in pediatric populations is less than that in adults. This in part may be because most regional anesthesia in children is performed under general anesthesia (which raises seizure threshold) using smaller local anesthetic volumes, and children likely have a lower comorbidity burden.

In summary, clinical registries have the capability of identifying infrequent events that occur in large populations, but often at the expense of detailed analysis. Of recent events that were reported in sufficient detail, $72 \%$ were considered major: 42 seizures and 8 cardiovascular (CV) presentations. ${ }^{11}$ Expectedly, minor events are less likely to be reported at all. These data support the assertion that LAST, while rare, remains a serious complication of local anesthetic use. Ultrasound guidance reduces the likelihood of any LAST event, yet seizure and/or cardiac arrest still occur at an estimated rate of $0.26 / 1000$ ultrasound-guided blocks. ${ }^{43}$

\section{Case Reports and Series}

Voluminous case reports published over the past decade emphasize the need to educate anesthesiologists and especially nonanesthesiologists in the prevention, recognition, and treatment of LAST. Case reports and case series allow in-depth analysis of individual incidents of LAST, but without the ability to gain insight into population-based frequency. The power of case report analysis is the ability to discern patient comorbidities, technique details, and treatment effectiveness, together with the ability to appreciate evolving trends in LAST presentation. The latter is important in assessing the impact of newer local anesthetic agents, nerve localization techniques (ultrasound guidance), and/or treatment modalities such as lipid emulsion therapy. Insights from case report analysis will be presented in Recognition section. 
In summarizing knowledge related to the incidence or frequency of LAST, we wish to emphasize that regardless of reporting mechanism, administrative data, registries, or case reports, the true frequency of LAST in the anesthesiology community is very likely underreported. This stems from a number of factors including misdiagnosis, publication bias, and individual practitioner underreporting, especially because it relates to nonanesthesiologists. The panel opines that, even if the rates of LAST are decreasing, the complication should not be trivialized. When placed into the perspective of other rare anesthetic complications, serious LAST occurs more frequently than epidural hematoma and at least as often as peripheral nerve injury, and is unique to both in its potential for mortality.

\section{Prevention}

Prevention is the primary and preferred mechanism for reducing the frequency and severity of LAST; meticulous attention to detail is the most important aspect of prevention. Optimal prevention is a multifactorial process, because no single intervention eliminates risk. Prevention involves 3 facets: avoidance and/or recognition of direct intravascular injection of local anesthetic, mitigating systemic uptake of local anesthetic from soft tissues, and awareness of patients at increased risk for LAST. The latter topic will be discussed in Recognition section.

\section{Limiting Local Anesthetic Uptake}

Table 2 reviews key prevention strategies. Avoidance of intravascular injection is best accomplished by ultrasound guidance, judicious use of an intravascular marker, and incremental injection of local anesthetic through needles and catheters based on an appropriate circulation time between injections (longer for lower extremity blocks and at-risk patients, such as those with slower circulation). Systemic uptake of local anesthetic is best mitigated by awareness of those regional techniques most associated with delayed systemic uptake, by prolonging drug clearance through the use of epinephrine, ${ }^{44}$ and by using the lowest effective local anesthetic dose, which can be facilitated by low volume ultrasoundguided techniques. ${ }^{43}$ The panel wishes to emphasize awareness that local anesthetic toxicity is additive - whether by subsequent redosing of the same or a different local anesthetic and whether administration of the drug is by the same or a different member of the perioperative team. The latter scenario may occur when anesthesiologist and surgeon both administer local anesthetic during the care of a patient.

\section{LAST and the Newer Regional Anesthetic Techniques}

New regional anesthetic techniques have expanded into anesthesiologists' practice. These include a variety of fascial plane-based truncal blocks, local infiltration anesthesia (LIA), and extended release liposomal bupivacaine (LB). Published information on these topics is limited, which impacts the panel's ability to offer definitive recommendations.

Within the anesthesiology community, the largest growth in new techniques involves truncal blocks that target fascial planes (eg, transversus abdominis plane, rectus sheath, quadratus lumborum, pectoral blocks). ${ }^{45}$ Within the surgical community, LIA has expanded, particularly in total joint arthroplasty practice. Case reports document LAST occurrences in both truncal block ${ }^{46}$ and LIA $^{47}$ patients. The former may be at particularly high risk for LAST because targeted tissue planes are very vascular, local anesthetic volumes are high, and the blocks are often used in at-risk

TABLE 2. Recommendations for Preventing LAST

- There is no single measure that can prevent LAST in clinical practice.

- Ultrasound guidance significantly reduces the risk of LAST in humans undergoing peripheral nerve block. Nevertheless, individual reports describe LAST despite the use of ultrasound. (I, B)

- Use the lowest effective dose of local anesthetic (dose $=$ product of volume $\times$ concentration). (I, C)

- Use incremental injection of local anesthetics - administer 3 to $5 \mathrm{~mL}$ aliquots, pausing 15-30 seconds between each injection. When using a fixed needle approach, eg, landmark, paresthesia-seeking, or electrical stimulation, time between injections should encompass 1 circulation time $(\sim 30-45 \mathrm{~s})$; however, this ideal may be balanced against the risk of needle movement between injections. Circulation time may be increased with lower extremity blocks or in those patients with diminished cardiac output. Use of larger dosing increments suggests the need for longer intervals to reduce the cumulative dose from stacked injections. (I, C)

- Aspirate the needle or catheter before each injection, recognizing that there is an $\sim 2 \%$ false negative rate for this diagnostic intervention. (I, $B$ )

-When injecting potentially toxic doses of local anesthetic, use of an intravascular marker is recommended. Although epinephrine is an imperfect maker and its use is open to physician judgment, its benefits likely outweigh its risks in most patients (IIa, B):

- Intravascular injection of epinephrine $10-15 \mu \mathrm{g} / \mathrm{mL}$ in adults produces a $\geq 10$ beat heart rate increase or a $\geq 15 \mathrm{~mm}$ Hg systolic blood pressure increase in the absence of $\beta$-blockade, active labor, advanced age, or general $/$ neuraxial anesthesia.

o Intravascular injection of epinephrine $0.5 \mu \mathrm{g} / \mathrm{kg}$ in children produces a $\geq 15 \mathrm{~mm} \mathrm{Hg}$ increase in systolic blood pressure.

- Appropriate subtoxic doses of local anesthetic can produce subjective symptoms of mild systemic toxicity (auditory changes, excitation, metallic taste, etc) in unpremedicated patients.

- Fentanyl $100 \mu \mathrm{g}$ produces sedation if injected intravascularly in laboring patients.

- Caregivers should be aware of the additive nature of local anesthetic toxicity and adjust accordingly local anesthetic redosing and/or administration by different perioperative providers. (II, B)

- The risk of LAST associated with truncal blocks may be reduced by using lower concentrations of local anesthetics, dosing on lean body weight, adjunctive epinephrine, and observation for at least 30 to 45 minutes after the block. (I, C)

- Patients receiving $L B$ should receive the same level of vigilance afforded to any patient receiving a local anesthetic. (I, C)

- Include local anesthetic dosing parameters and at-risk patient concerns as part of the preincisional surgical pause. (I, C)

These recommendations are intended to encourage optimal patient care but cannot ensure the avoidance of adverse outcomes. As with any practice advisory recommendation, these are subject to revision as knowledge advances regarding specific complications.

The class of recommendation and level of evidence for each intervention are given in parenthesis (see Table 1).

Changes from the 2010 LAST practice advisory $^{1}$ are italicized. 
patient populations, that is, children and parturients. The advisory panel agrees with the recommendations put forth by Chin et $\mathrm{al}^{48}$ in their recent review of abdominal wall blocks. Their recommended strategies to reduce the likelihood of LAST include using epinephrine to reduce local anesthetic bioavailability, ${ }^{49}$ using dilute concentrations of less cardiotoxic local anesthetics, basing dose calculations on lean body weight, and 30- to 45-minute monitoring periods to account for the prolonged time to local anesthetic peak plasma concentration that is inherent to truncal blocks. $^{48}$ (IIa; B)

Data pertinent to LB and LAST are limited, which is both reassuring and worthy of conservative interpretation. Ilfeld et al's ${ }^{50}$ retrospective review of 6 published RCTs of LB use for 335 PNBs reported no incident of identifiable LAST. Incidents of dose-related bradycardia $(2 \%-14 \%)$ were conceivably consistent with limited LAST, but by no means diagnostic. To our knowledge, there are no published case reports of LAST associated with LB (possibly indicating negative reporting bias for adverse events); however, 130 cases (January 2012 to June 2016) are present in the US Food and Drug Administration's (FDA's) Adverse Event Reporting System. This voluntary self-reporting system, which does not have a code specific to LAST, is non-peer reviewed and is open to reports from medical professionals, industry, and private individuals. Using the statistical technique of disproportionality analysis, Aggarwal ${ }^{51}$ reports an association between LB and LAST based on the FDA's Adverse Event Reporting System data.

The corpus of published human studies on LB totals only a few thousand patients, the drug has been clinically available in the United States for only 5 years, and the pharmacologic and chemical dynamics of liposomal formulations are dramatically different from standard local anesthetics. Based on such limited information, our recommendation is that patients receiving LB be afforded the same level of vigilance as would be given to any patient receiving a local anesthetic (IIb; C).

\section{Prevention Strategies}

In the interval since our second practice advisory, 2 new findings related to prevention deserve emphasis. First is the evolving role of ultrasound guidance. Meta-analysis had shown that ultrasound reduced the incidence of vascular puncture associated with PNB compared with peripheral nerve stimulation, ${ }^{52}$ yet previous institutional ${ }^{32,36}$ and registry ${ }^{39}$ data did not link this surrogate outcome to a definitive reduction in seizure or cardiac arrest. In the intervening years, Barrington and Kluger ${ }^{35}$ have confirmed that ultrasound guidance reduces the incidence of LAST episodes spanning the continuum from mild subjective symptoms of toxicity to seizure and cardiac arrest. As compared with landmark techniques, ultrasound reduced the risk of LAST by $65 \%$ in their study of over 25,000 PNBs. These findings are consistent with those reported by Orebaugh et al. ${ }^{37}$ The second new finding related to prevention involves the changing patterns of LAST presentation, as discussed below.

\section{Recognition: The Changing Patterns of LAST Presentation}

Our 2010 advisory was accompanied by DiGregorio et al's ${ }^{4}$ analysis of 93 LAST cases reported over the 30-year period, 1979 to $2009{ }^{4}$ In the interim, Vasques et $\mathrm{l}^{53}$ presented 67 additional cases from 2010 to March 2014. Accompanying the current advisory, Gitman and Barrington ${ }^{11}$ present 47 more cases reported between April 2014 and November 2016. These case series span nearly 4 decades and serve to confirm some expected patterns of LAST clinical presentation while revealing changing patterns in others. First, all 3 case series reinforce the concept that LAST events are skewed toward the extremes of age. The recent finding that large amounts of absorbed local anesthetic are stored in skeletal muscle suggests that patients with low muscle mass are at higher risk for LAST. The most recent Pediatric Regional Anesthesia Network data confirm similar observations in infants under 6 months old, whose rate of serious LAST is 6-fold higher than that for other children (J.M. Neal, MD, and D.M. Polaner, MD, personal written communication on September 19, 2017). In addition to lower muscle mass, infants and neonates manifest a higher unbound fraction of local anesthetic. Second, although bupivacaine is the most toxic local anesthetic and the most difficult overdose to resuscitate when LAST occurs, all local anesthetics are capable of causing toxicity, including "safer" agents such as lidocaine and ropivacaine (26\% and $21 \%$, respectively, of total events in the latest case series). ${ }^{11}$ The advisory panel and others ${ }^{35,54}$ speculate that practitioners may be less vigilant with regard to dosing and safety steps when administering these purportedly less toxic local anesthetics. Furthermore, the panel opines that, when viewed on an equipotent basis, ropivacaine as administered in most clinical settings is likely similar in toxicity to bupivacaine. Third, about $15 \%$ of LAST events involve continuous local anesthetic infusions and most episodes present 1 to 4 days after initiation of the infusion, often with subtle prodromes or hemodynamic changes.

The combined case series ${ }^{4,11,53}$ also reveal shifting patterns in LAST's clinical presentation. First, an increasing number of events (about 20\%) occur outside of the traditional hospital environment and half involve a nonanesthesiologist. The occurrence of LAST after simple tissue infiltration of local anesthetic in $20 \%$ of events is consistent with nonanesthesia-trained practitioners administering the drug and/or an increase in LIA techniques. Similar events involve topical anesthesia of the airway or transcutaneous absorption of local anesthetics. Second, a new signal from the current case series ${ }^{11}$ involves a preponderance of pediatric LAST events associated with penile block, which accounted for a quarter of case reports during that series' 2.5 -year period. Some of these cases required chest compression. ${ }^{55}$ Encouragingly, after implementing policy, dosing, and technique changes, the involved institution reports no further LAST events associated with penile block (G. Weinberg, MD and R. Yu, MD, personal verbal communication May 2017).

The 2010 practice advisory noted that $40 \%$ of reported cases did not present in a manner consistent with the classic textbook description of LAST. Rather than an initial complex of CNS excitement, for example, metallic taste or auditory changes, followed by seizure and then CV collapse, nearly half of the presentations were considered "atypical," presenting with CV signs alone (no evidence of CNS toxicity) and/or having a delayed presentation (more than 5 minutes after injection of local anesthetic). Although the occurrence rates vary slightly, the interim analyses from 2010 to $2016^{11,53}$ conform to previous observations that about one third of patients present with combined CNS/CV signs and symptoms (mostly seizures, hypertension or hypotension, and electrocardiographic changes). Fewer patients exhibit isolated CV symptoms as compared with isolated CNS symptoms (Figs. 2, 3). Another change to clinical presentation is a shift in timing from signs and symptoms appearing coincident with or within a minute of local anesthetic administration (intravascular injection) to presentations that are delayed from several minutes to 30 or even 60 minutes after injection ${ }^{11,53}$ (Fig. 4). We speculate that this shift toward delayed presentation involves fewer intravascular injections as a consequence of ultrasound guidance, the increasing use of local tissue infiltration techniques, and/or increasing use of continuous local anesthetic infusion. This shift strengthens our previous advice to observe patients for at least 30 minutes after injecting potentially toxic doses of local anesthetic. 


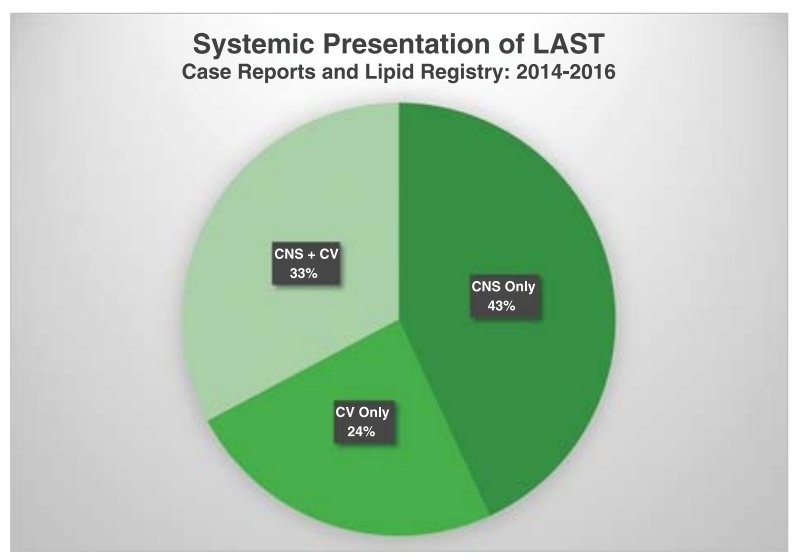

FIGURE 2. Local anesthetic systemic toxicity presentation by organ system, as described in case reports from 2014 to 2016 . $^{11}$

Despite changing patterns in LAST presentation, our recommendations remain consistent. Local anesthetic systemic toxicity is a masquerader, and its detection requires persistent open-minded vigilance. To emphasize reasonable local anesthetic dosing and to highlight those patients most at risk for LAST, we recommend including a discussion of local anesthetic use during the preincisional surgical pause. Doing so facilitates awareness among all perioperative

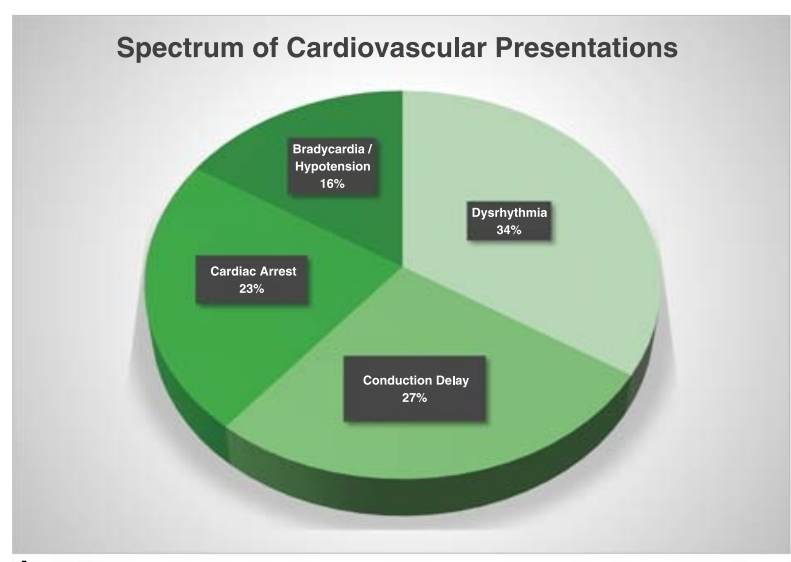

A

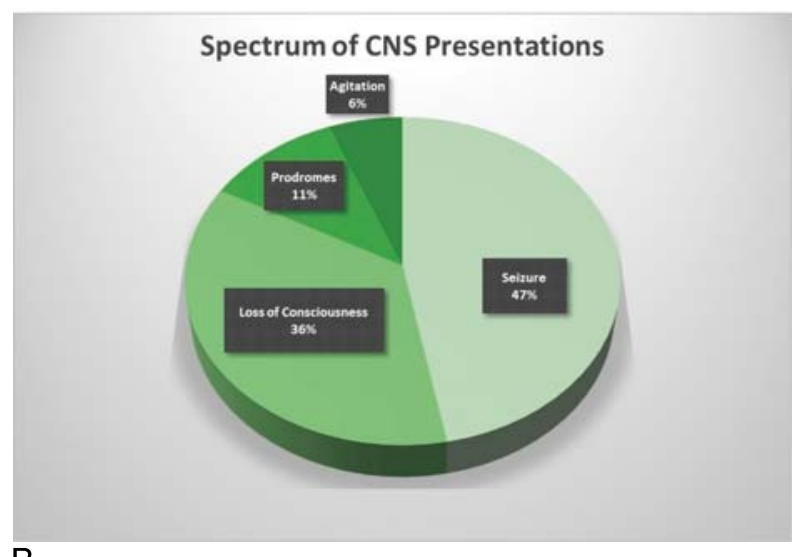

B

FIGURE 3. Spectrum of LAST clinical presentations, as described in case reports from 2014 to $2016 .^{11}$

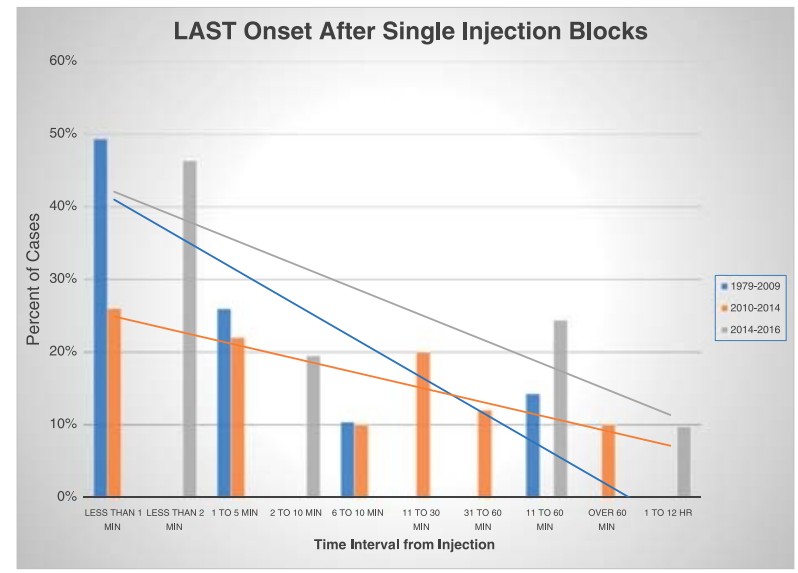

FIGURE 4. The changing time intervals between injection of local anesthetic and initial presentation of LAST over 3 time periods: 1979 to $2009,{ }^{4} 2010$ to $2014,^{53}$ and 2014 to $2016 .{ }^{11}$ The changing slopes of trend lines suggest that contemporary LAST presentations are becoming more delayed as compared with previous years.

team members of maximal local anesthetic dosing and the issue of additive toxicity, particularly because it pertains to local anesthetic administration by different team members, including nonanesthesiologists. The surgical pause is also an opportunity to highlight at-risk patients and how they might present with LAST. Classic presentation patterns that involve subjective signs, seizure, or cardiac arrest in close proximity to local anesthetic dosing do not always occur. When patients receive a local anesthetic and manifest a delayed presentation of agitation or CNS depression, or unexplained signs of cardiac compromise such as progressive hypotension or bradycardia, LAST should be considered in the differential diagnosis. Table 3 outlines factors believed to heighten the risk of LAST. Table 4 reviews recommendations for diagnosing LAST.

\section{Treatment: Evolving Paradigms}

The treatment of severe LAST is fundamentally different from conventional cardiopulmonary resuscitation (CPR) insofar as toxic cardiomyopathy differs pathophysiologically from other causes of CV collapse. First, unlike contemporary CPR paradigms that eschew early respiratory interventions in favor of cardiac support, successful treatment of LAST hinges on the primacy of airway management to prevent the hypoxia, hypercapnia, and acidosis that potentiate LAST and negatively impact resuscitative efforts. The mechanism of potentiation may be related to increased free fraction of local anesthetic and/or worsening of cardiac function. Second, while CPR and Advanced Cardiac Life Support (ACLS) symptomatically treat hemodynamic perturbations consequent to ischemic cardiac injury, usually with the goal of improving coronary perfusion, successful treatment of LAST seeks to effectively moderate or reverse the mechanisms underlying the local anesthetic toxicity. Recent evidence indicates that the inotropic benefit of lipid only occurs when myocardial local anesthetic content is reduced below a threshold that corresponds to ion channel blocking concentrations. This underlines the importance of effective CPR to ensure that coronary perfusion is sufficient to reduce local anesthetic tissue levels to attain the maximum benefit of lipid infusion. Third, recent data from rat models reinforce the admonition that epinephrine, when necessary, be administered initially in small doses ( $1 \mu \mathrm{g} / \mathrm{kg}$ or less) to avoid impaired 


\section{TABLE 3. Risk Factors for LAST}

- Patient characteristics

- Extremes of age- - less than 16 or more than 60 years

○ Low muscle mass - particularly with neonates, infants, and the debilitated elderly

$\circ$ Female $>$ male

$\circ$ Comorbidities

- Cardiac disease, especially arrhythmias, conduction abnormalities, ischemia, and congestive heart failure

- Liver disease

- Metabolic disease, especially diabetes mellitus, isovaleric acidemia, mitochondrial disease, and carnitine deficiency

- CNS diseases

- Low plasma protein binding - liver disease, malnourishment, infants, pregnancy

- Local anesthetic characteristics

- Bupivacaine has a lower safety margin and resuscitation is more difficult in the event of LAST, but local anesthetics such as ropivacaine and lidocaine still account for a significant proportion of LAST events.

- Block site, total local anesthetic dose, test dosing, and patient comorbidities are more predictive of high plasma levels of local anesthetic than are body weight or body mass index.

- Local anesthetic infusions are particularly problematic after 1 to 4 days and in patients of small body mass.

- Seizure is up to 5 times more likely after PNB than epidural block.

- Practice setting

○ Up to $20 \%$ of LAST cases occur outside of the hospital setting.

○ Nonanesthesiologists are involved in up to $50 \%$ of LAST cases

Risk factors are extrapolated primarily from case reports and series. This table is derived from mostly IIa; B evidence.

pulmonary gas exchange ${ }^{56}$ and increased afterload. Fourth, simulated LAST scenarios have demonstrated the usefulness of the ASRA Checklist ${ }^{57}$ as a cognitive aid during LAST treatment ${ }^{58}$ and the value of a reader to prompt adherence to recommendations. ${ }^{59}$ An updated version of the ASRA Checklist is part of this practice advisory series. ${ }^{13}$ The ASRA LAST smart phone app (iOS and Android) has been updated coincident with this practice advisory.

\section{Insights Regarding Contemporary LAST Treatment}

The case report analysis presented with this practice advisory ${ }^{11}$ allows insights into the contemporary treatment of LAST.
Treatment protocols developed by national organizations such as ASRA $^{57}$ and the Association of Anaesthetists of Great Britain and Ireland ${ }^{60}$ are followed inconsistently. Less than half (47\%) of patients with LAST were treated with lipid emulsion, and nearly $40 \%$ of those patients received the antidote 10 minutes or longer after symptom onset, occasionally at an incorrect dose. Nevertheless, and within the limits of case series comparison, the overall use of lipid emulsion has apparently increased from 34\% to $47 \%$ as compared with our 2010 report. ${ }^{4}$ An ACLS was necessary in $21 \%$ of patients; duration ranged from 2 to 60 minutes. Two of the reported 47 patients died; one from a combination of delayed diagnosis and treatment, and the other self-administered over $4 \mathrm{~g}$

TABLE 4. Recommendations for Diagnosing LAST

- Classic descriptions of LAST depict a progression of subjective symptoms of CNS excitement (agitation, auditory changes, metallic taste or abrupt onset of psychiatric symptoms), followed by seizures then CNS depression (drowsiness, coma, or respiratory arrest). Near the end of this continuum, initial signs of cardiac toxicity (hypertension, tachycardia, or ventricular arrhythmias) are supplanted by cardiac depression (bradycardia, conduction block, asystole, decreased contractility, and hypotension). However, there is substantial variation in this classic description, including:

- Simultaneous presentation of CNS and cardiac toxicity

- Cardiac toxicity without prodromal signs and symptoms of CNS toxicity

○ Thus, the practitioner must be vigilant for atypical or unexpected presentation of LAST. (I; B)

- The timing of LAST presentation is variable. Immediate $(<60 \mathrm{~s})$ presentation suggests intravascular injection of local anesthetic with direct access to the brain, whereas presentation that is delayed 1 to 5 min suggests intermittent intravascular injection, lower extremity injection, or delayed tissue absorption. Recent case reports suggest a shift toward delayed presentations of LAST. Because LAST can present more than 15 min and occasionally over an hour after injection, patients who receive potentially toxic doses of local anesthetic should be closely monitored for at least 30 min after injection. (I; B)

- The overall variability of LAST signs and symptoms, timing of onset, and association with various disease states suggests that practitioners should maintain a low threshold for considering the diagnosis of LAST in patients with atypical or unexpected presentation of CNS or cardiac signs and symptoms after receiving more than a minimal dose of local anesthetic. (IIa; B)

These recommendations are intended to encourage optimal patient care but cannot ensure the avoidance of adverse outcomes. As with any practice advisory recommendation, these are subject to revision as knowledge advances regarding specific complications.

The class of recommendation and level of evidence for each intervention are given in parenthesis (see Table 1).

Changes from the 2010 LAST practice advisory ${ }^{1}$ are italicized. 
of topical lidocaine at home. As expected, all 11 unpublished cases submitted to lipidrescue.org from April 2014 to November 2016 received lipid emulsion immediately or within 5 minutes of symptom onset. Three of these patients required ACLS, but none died.

\section{New Treatment Recommendations}

The use of lipid emulsion as an antidote for LAST is "off label," as defined by the FDA. Our 2010 advisory suggested using lipid emulsion therapy at the first sign of arrhythmia, prolonged seizures, or rapid clinical deterioration of the patient. Based on evidence that lipid shuttling is most effective early in the toxic event ${ }^{10}$ when local anesthetic plasma concentrations are at their peak, we now unequivocally recommend lipid emulsion therapy soon after airway management in any LAST event that is judged to be potentially serious. Furthermore, because adverse effects from lipid administration specifically as an antidote for LAST appear to be minor and limited, we recommend erring on the early side of administration. The maximum lipid dose has been increased to $\sim 12 \mathrm{~mL} / \mathrm{kg}$ based on FDA recommendations. ${ }^{61}$ Nevertheless, total lipid doses used in resuscitation of LAST are usually much less (typically about half) of this limit. Thus, it is important to avoid delivering an excessive dose of lipid since dangerous fat overload can occur. ${ }^{62}$ Table 5 outlines treatment recommendations.

\section{Future Directions}

If the previous decade is any indication, we expect continued progress in our understanding of LAST - its incidence, cellular mechanisms, prevention, and treatment. Much work remains with regard to understanding the cellular and systemic mechanisms inherent to local anesthetic poisoning. Advancement of this knowledge holds promise for improved resuscitation. Whether or not the overall incidence of LAST is truly diminishing remains open to debate, particularly because it pertains to the healthcare community at large, in nonhospital settings, and among nonanesthesiologist practitioners. Hopefully the future will see further development of local anesthetic alternatives such as neosaxitoxin, which blocks

\section{TABLE 5. Recommendations for Treatment of LAST}

- If signs and symptoms of LAST occur, prompt and effective airway management is crucial to preventing hypoxia, hypercapnia, and acidosis, which are known to potentiate LAST. (I; B)

- Lipid emulsion therapy $(I ; B)$ :

- Administer at the first signs of LAST, after airway management

- Timeliness of lipid emulsion is more important than the order of administration modality (bolus vs infusion)

- $20 \%$ lipid emulsion BOLUS

- $100 \mathrm{~mL}$ over 2-3 min if patient is over $70 \mathrm{~kg}$

- $1.5 \mathrm{~mL} / \mathrm{kg}$ over 2-3 min if patient is less than $70 \mathrm{~kg}$

- $20 \%$ lipid emulsion INFUSION

-200-250 mL over 15-20 min if patient is over $70 \mathrm{~kg}$

- $0.25 \mathrm{~mL} / \mathrm{kg} / \mathrm{min}$ if patient is less than $70 \mathrm{~kg}$ (ideal body weight)

- If circulatory stability is not attained, consider rebolus or increasing infusion to $0.5 \mathrm{~mL} / \mathrm{kg} / \mathrm{min}$

- Continue infusion for at least $10 \mathrm{~min}$ after circulatory stability is attained.

- Approximately $12 \mathrm{~mL} / \mathrm{kg}$ lipid emulsion is recommended as the upper limit for initial dosing. (IIb; B)

- Propofol is not a substitute for lipid emulsion. (III; B)

- Seizure control:

- If seizures occur, they should be rapidly halted with benzodiazepines. If benzodiazepines are not readily available, lipid emulsion or small doses of propofol are acceptable. (I; B)

- Although propofol can stop seizures, large doses further depress cardiac function; propofol should be avoided when there are signs of cardiovascular compromise. (III; B)

- If seizures persist despite benzodiazepines, small doses of succinylcholine or similar neuromuscular blocker should be considered to minimize acidosis and hypoxemia. (I; C)

- If cardiac arrest occurs:

- If epinephrine is used, small initial doses $(\leq 1 \mu \mathrm{g} / \mathrm{kg})$ are preferred. (IIa; $B)$

- Vasopressin is not recommended. (III; B)

- Avoid calcium channel blockers and $\beta$-adrenergic receptor blockers. (III; C)

o If ventricular arrhythmias develop, amiodarone is preferred (IIa; B); treatment with local anesthetics (lidocaine or procainamide) is not recommended. (III; B)

- Failure to respond to lipid emulsion and vasopressor therapy should prompt institution of CPB $(I ; B)$. Because there can be considerable lag in beginning $\mathrm{CPB}$, it is reasonable to notify the closest facility capable of providing it when cardiovascular compromise is first identified during an episode of LAST.

- Patients with a significant CVevent should be monitored for at least 4-6 h. If the event is limited to CNS symptoms that resolve quickly, they should be monitored for at least $2 \mathrm{~h}$. (IIa; $B$ )

- Use written or electronic checklists as cognitive aids during the management of LAST. A dedicated reader improves adherence to the checklist. (I; A)

These recommendations are intended to encourage optimal patient care but cannot ensure the avoidance of adverse outcomes. As with any practice advisory recommendation, these are subject to revision as knowledge advances regarding specific complications.

The class of recommendation and level of evidence for each intervention are given in parenthesis (see Table 1).

Changes from the 2010 LAST practice advisory ${ }^{1}$ are italicized.

CPB indicates cardiopulmonary bypass. 
sensory conduction by binding to the external pores of noncardiac sodium channels and does not cross the blood-brain barrier. ${ }^{63}$ Neosaxitoxin thus prolongs anesthesia without the risk of local anesthetic-induced cardio- and CNS toxicity. Until such time as alternative drugs come into use, the burden is on anesthesiologists and LAST researchers to improve our knowledge of basic mechanisms, enhance early recognition, improve awareness, and build effective systems for prevention and treatment.

To reiterate our concluding comments from the 2010 practice advisory, "LAST remains a significant clinical problem. Considering 1) the extensive use of local anesthetics, 2) the frequent use of doses sufficient to cause significant morbidity or mortality, and 3) the imperfect nature of our ability to predict, prevent, detect, and treat these complications, it remains the responsibility of all clinicians using local anesthetics to understand their potential for causing severe systemic toxicity and to be prepared to respond immediately when these events occur." "Moreover, subtle or undetected patient comorbidities can significantly increase susceptibility to LAST. Practice patterns and system improvements may well reduce the likelihood of LAST and optimize patient safety. Nevertheless, although we contend that the frequency of LAST can be reduced, it is unlikely to be eliminated altogether - there will always be system errors, unrecognized patient comorbidities, and stochastic events that invariably arise in large populations. Although LAST is indeed a rare event, it still occurs despite proper technique used by competent professionals. When LAST happens, recognition and preparedness make it a treatable complication of regional anesthesia.

\section{ACKNOWLEDGMENTS}

The authors thank the following colleagues who made substantial contributions to this project through authorship of the individual background articles from which this executive summary was drawn: Hospital for Special Surgery, Weill Cornell Medical College, New York, NY: Crispiana Cozowicz, MD; Mt. Sinai School of Medicine, New York, NY: Nicole Zubizarreta, MPH and Jashvant Poeran, MD, PhD; University of Illinois College of Medicine, Chicago, IL: Steven Roth, MD; Pritzker School of Medicine, University of Chicago, Chicago, IL: Monica Matsumoto, BA; Stanford University, Palo Alto, CA: T. Kyle Harrison, MD; and Virginia Mason Medical Center, Seattle, WA: Crystal M. Woodward, MD.

\section{REFERENCES}

1. Neal JM, Bernards CM, Butterworth JF, et al. ASRA practice advisory on local anesthetic systemic toxicity. Reg Anesth Pain Med. 2010; 35:152-161.

2. Corcoran W, Butterworth J, Weller RS, et al. Local anesthetic-induced cardiac toxicity: a survey of contemporary practice strategies among academic anesthesiology departments. Anesth Analg. 2006;103: 1322-1326.

3. Butterworth JF 4rh. Models and mechanisms of local anesthetic cardiac toxicity: a review. Reg Anesth Pain Med. 2010;35:167-176.

4. Di Gregorio G, Neal JM, Rosenquist RW, Weinberg GL. Clinical presentation of local anesthetic systemic toxicity: a review of published cases, 1979 to 2009. Reg Anesth Pain Med. 2010;35:181-187.

5. Drasner K. Local anesthetic systemic toxicity: a historical perspective Reg Anesth Pain Med. 2010;35:160-164

6. Mulroy MF, Hejtmanek MR. Prevention of local anesthetic systemic toxicity. Reg Anesth Pain Med. 2010;35:177-180.

7. Weinberg GL. Treatment of local anesthetic systemic toxicity (LAST) Reg Anesth Pain Med. 2010;35:188-193.

8. Kahn R, Gale EA. Gridlocked guidelines for diabetes. Lancet. 2010;375 2203-2204
9. Mörwald EE, Zubizarreta N, Cozowicz C, Poeran J, Memtsoudis SG Incidence of local anesthetic systemic toxicity in orthopedic patients receiving peripheral nerve blocks. Reg Anesth Pain Med. 2017;42: $442-445$

10. Fettiplace MR, Weinberg G. The mechanisms underlying lipid resuscitation therapy. Reg Anesth Pain Med. 2018;43:138-149.

11. Gitman M, Barrington MJ. Local anesthetic systemic toxicity: a review of recent case reports and registries. Reg Anesth Pain Med. 2018;43: $124-130$

12. Rubin DS, Motsumoto M, Weinberg G, Roth S. Local anesthetic systemic toxicity in total joint arthroplasty: incidence and risk factors in the United States from the National Inpatient Sample 1998-2013. Reg Anesth Pain Med. 2018;43:131-137.

13. Neal JM, Woodward CM, Harrison TK. The American Society of Regional Anesthesia and Pain Medicine checklist for managing local anesthetic systemic toxicity: 2017 version. Reg Anesth Pain Med. 2018;43:150-153.

14. Eagle KA, Berger PB, Calkins $\mathrm{H}$, et al. ACC/AHA guideline update for perioperative cardiovascular evaluation for noncardiac surgery: executive summary: a report of the American College of Cardiology/American Heart Association Task Force on Practice Guidelines (Committee to Update the 1996 Guidelines on Perioperative Cardiovascular Evaluation for Noncardiac Surgery). J Am Coll Cardiol. 2002;39:542-553.

15. ASA Standards, Guidelines, Statements and Practice Parameters Available at: http://www.asahq.org/resources/clinical-information. Accessed January 21, 2015

16. Neal JM, Kopp SL, Lanier WL, Pasternak JJ, Rathmell JP. Anatomy and pathophysiology of spinal cord injury associated with regional anesthesia and pain medicine: 2015 update. Reg Anesth Pain Med. 2015; 40:506-525

17. Weinberg GL, VadeBoncouer TR, Ramaraju GA, Garcia-Amaro MF, Cwik MJ. Pretreatment or resuscitation with a lipid emulsion shifts the dose-response to bupivacaine-induced asystole in rats. Anesthesiology. 1998;88:1071-1075.

18. Fettiplace MR, Lis K, Ripper R, et al. Multi-modal contributions to detoxification of acute pharmacotoxicity by a triglyceride micro-emulsion. J Control Release. 2015;198:62-70.

19. Dix SK, Rosner GF, Nayar M, et al. Intractable cardiac arrest due to lidocaine toxicity successfully resuscitated with lipid emulsion. Crit Care Med. 2011;39:872-871.

20. Dureau P, Charbit B, Nicolas N, Benhamou D, Mazoit JX. Effect of Intralipid $^{\circledR}$ on the dose of ropivacaine or levobupivacaine tolerated by volunteers. Anesthesiology. 2016;125:474-483.

21. Shi K, Xia Y, Wang Q, et al. The effect of lipid emulsion on pharmacokinetics and tissue distribution of bupivacaine in rats. Anesth Analg. 2013;116:804-809.

22. Heinonen JA, Litonius E, Salmi T, et al. Intravenous lipid emulsion given to volunteers does not affect symptoms of lidocaine brain toxicity. Basic Clin Pharmacol Toxicol. 2015;116:378-383.

23. Fettiplace MR, Ripper R, Lis K, et al. Rapid cardiotonic effects of lipid emulsion infusion. Crit Care Med. 2013;41:e156-e162.

24. Stehr SN, Ziegeler JC, Pexa A, et al. The effects of lipid infusion on myocardial function and bioenergetics in 1-bupivacaine toxicity in the isolated rat heart. Anesth Analg. 2007;104:186-192.

25. Fettiplace MR, Kowal K, Ripper R, et al. Insulin signaling in bupivacaine-induced cardiac toxicity: sensitization during recovery and potentiation by lipid emulsion. Anesthesiology. 2016;124: 428-442.

26. Lou P-H, Lucchinett E, Zhang L, et al. The mechanism of Intralipid®mediated cardioprotection complex IV inhibition by the active metabolite, palmitoylcarnitine, generates reactive oxygen species and activates reperfusion injury salvage kinases. PLoS One. 2014; 9:e87205. 
27. Rahman S, Li J, Bopassa JCJ, et al. Phosphorylation of GSK-3b mediates intralipid-induced cardioprotection against Ischemia/reperfusion injury. Anesthesiology. 2011;115:242-253.

28. Mulroy MF. Systemic toxicity and cardiotoxicity from local anesthetics: Incidence and preventive measures. Reg Anesth Pain Med. 2002;27: 556-561.

29. Liu SS, Ortolan S, Sandoval MV, et al. Cardiac arrest and seizures caused by local anesthetic systemic toxicity after peripheral nerve blocks: Should we still fear the reaper? Reg Anesth Pain Med. 2016;41:5-21.

30. Weinberg G, Barron G. Local anesthetic systemic toxicity (LAST): Not gone, hopefully not forgotten. Reg Anesth Pain Med. 2016;41:1-2.

31. Premier Perspective Database. Available at: https://www.premierinc.com/ transforming-healthcare/healthcare-performanceimprovement/premierresearch-services/. Accessed June 13, 2016.

32. Sites BD, Taenzer AH, Herrick MD, et al. Incidence of local anesthetic systemic toxicity and postoperative neurologic symptoms associated with 12,668 ultrasound-guided nerve blocks. An analysis from a prospective clinical registry. Reg Anesth Pain Med. 2012;37:478-482.

33. Heinonen J, Litonius E, Pitkanen M, Rosenberg P. Incidence of severe local anaesthetic toxicity and adoption of lipid rescue in Finnish anaesthesia departments in 2011-2013. Acta Anaesthesiol Scand. 2015;59:1032-1037.

34. Rohrbaugh M, Kentor ML, Orebaugh SL, Williams B. Outcomes of shoulder surgery in the sitting position with interscalene nerve block: a single-center series. Reg Anesth Pain Med. 2013;38:28-33.

35. Barrington MJ, Kluger R. Ultrasound guidance reduces the risk of local anesthetic systemic toxicity following peripheral nerve blockade. Reg Anesth Pain Med. 2013;38:289-299.

36. Orebaugh SL, Williams BA, Vallejo M, Kentor ML. Adverse outcomes associated with stimulator-based peripheral nerve blocks with versus without ultrasound visualization. Reg Anesth Pain Med. 2009;34:251-255.

37. Orebaugh SL, Kentor ML, Williams BA. Adverse outcomes associated with nerve stimulator-guided and ultrasound-guided peripheral nerve blocks by supervised trainees: update of a single-site database. Reg Anesth Pain Med. 2012;37:577-582.

38. Allegri M, Bugada D, Grossi P, et al. Italian registry of complications associated with regional anesthesia (RICALOR). An incidence analysis from a prospective clinical survey. Minerva Anestesiol. 2016;82:392-402.

39. Barrington MJ, Watts SA, Gledhill SR, et al. Preliminary results of the Australasian Regional Anaesthesia Collaboration: a prospective audit of more than 7000 peripheral nerve and plexus blocks for neurologic and other complications. Reg Anesth Pain Med. 2009;34:534-541.

40. Ecoffey C, Lacroix F, Giaufré E, Orliaguet G, Courrèges P. Epidemiology and morbidity of regional anesthesia in children: a follow-up one-year prospective survey of the French-Language Society of Paediatric Anaesthesiologists (ADARPEF). Paediatr Anaesth. 2010;20:1061-1069.

41. Gurnaney H, Kraemer FW, Maxwell L, Muhly WT, Schleelein L, Ganesh A. Ambulatory continuous peripheral nerve blocks in children and adolescents: a longitudinal 8-year single center study. Anesth Analg. 2014; 118:621-627.

42. Polaner DM, Taenzer AH, Walker BJ, et al. Pediatric Regional Anesthesia Network (PRAN): a multi-institutional study of the use and incidence of complications of pediatric regional anesthesia. Anesth Analg. 2012;115 1353-1364.

43. Neal JM. Ultrasound-guided regional anesthesia and patient safety: update of an evidence-based analysis. Reg Anesth Pain Med. 2016;41:195-204.

44. Bernards CM, Kopacz DJ. Effect of epinephrine on lidocaine clearance in vivo: a microdialysis study in humans. Anesthesiology. 1999;91:962-968.

45. Tran DQ, Boezaart AP, Neal JM. Beyond ultrasound guidance for regional anesthesiology. Reg Anesth Pain Med. 2017;42:556-563.
46. Weiss E, Jolly C, Dumoulin JL, et al. Convulsions in 2 patients after bilateral ultrasound-guided transversus abdominis plane blocks for Cesarean analgesia. Reg Anesth Pain Med. 2014;39:248-251.

47. Fenten MG, Rohrbach A, Wymenga AB, Stienstra R. Systemic local anesthetic toxicity after local infiltration analgesia following a polyethylene tibial insert exchange: a case report. Reg Anesth Pain Med. 2014;39: 264-265.

48. Chin KJ, McDonnell JG, Carvalho B, Sharkey A, Pawa A, Gadsden J. Essentials of our current understanding: abdominal wall blocks. Reg Anesth Pain Med. 2017;42:133-183.

49. Miranda P, Corvetto MA, Altermatt FR, Araneda A, Echevarria GC, Cortinez LI. Levobupivacaine absorption pharmacokinetics with and without epinephrine during TAP block: analysis of doses based on the associated risk of local anaesthetic toxicity. Eur J Clin Pharmacol. 2016; 72:1221-1227.

50. Ilfeld BM, Viscusi ER, Hadzic A, et al. Safety and side effect profile of liposome bupivacaine (Exparel) in peripheral nerve blocks. Reg Anesth Pain Med. 2015;40:572-582.

51. Aggarwal N. Local anesthetics systemic toxicity association with Exparel (bupivacaine liposome) - a pharmacovigilance evaluation. Expert Opin Drug Saf. 2017;5:1-7.

52. Abrahams MS, Aziz MF, Fu RF, Horn JL. Ultrasound guidance compared with electrical neurostimulation for peripheral nerve block: a systematic review and meta-analysis of randomized controlled trials. Br J Anaesth. 2009; 102:408-417.

53. Vasques F, Behr AU, Weinberg G, Ori C, Di Gregorio G. A review of local anesthetic systemic toxicity cases since publication of the American Society of Regional Anesthesia recommendations. To whom it may concern. Reg Anesth Pain Med. 2015;40:698-705.

54. Neal JM. Local anesthetic systemic toxicity. Improving patient safety one step at a time. Reg Anesth Pain Med. 2013;38:259-261.

55. Yu RN, Houck CS, Casta A, Blum RH. Institutional policy changes to prevent cardiac toxicity associated with bupivacaine penile blockade in infants. A A Case Rep. 2016;7:71-75.

56. Wang Q-G, Wu C, Xia Y, et al. Epinephrine deteriorates pulmonary gas exchange in a rat model of bupivacaine-induced cardiotoxicity: a threshold dose of epinephrine. Reg Anesth Pain Med. 2017;42:432-350.

57. Neal JM, Mulroy MF, Weinberg GL. American Society of Regional Anesthesia and Pain Medicine checklist for managing local anesthetic systemic toxicity: 2012 version. Reg Anesth Pain Med. 2012;37:16-18.

58. Neal JM, Hsiung RL, Mulroy MF, Halpern BB, Dragnich AD, Slee AE. ASRA checklist improves trainee performance during a simulated episode of local anesthetic systemic toxicity. Reg Anesth Pain Med. 2012;37:8-15.

59. McEvoy MD, Hand WR, Stoll WD, Furse CM, Nietert PJ. Adherence to guidelines for the management of local anesthetic systemic toxicity is improved by an electronic decision support tool and designated "reader". Reg Anesth Pain Med. 2014;39:299-305.

60. AAGBI Safety Guideline: Management of Severe Local Anaesthetic Toxicity. Association of Anaesthetists of Great Britain and Ireland. Available at: http://www.aagbi.org/sites/default/files/la_toxicity_2010_0. pdf. Accessed January 4, 2017.

61. Reference ID: 3958413 . US Food and Drug Administration. Available at: https://www.accessdata.fda.gov/drugsatfda_docs/label/2016/2076481bl. pdf. Accessed August 7, 2017.

62. Fettiplace MR, Akpa BS, Rubinstein I, Weinberg G. Confusion about infusion: rational volume limits for intravenous lipid emulsion during treatment of oral overdoses. Ann Emerg Med. 2015;66:185-188.

63. Templin JS, Wylie MC, Kim JD, et al. Neosaxitoxin in rat sciatic block: Improved therapeutic index using combinations with bupivacaine, with and without epinephrine. Anesthesiology. 2015;123:886-898. 